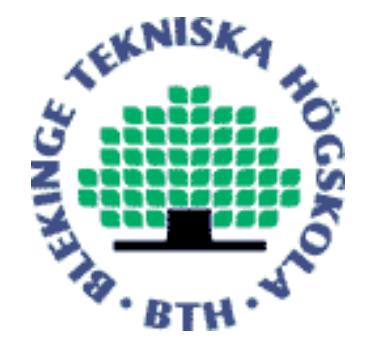

Copyright (C) 2014 IEEE.

Citation for the published paper:

Wireless Information and Power Transfer in an Underlay Cognitive Radio Network

Louis Sibomana, Hans-Jürgen Zepernick, Tran Hung

IEEE International Conference on Signal Processing and Communication Systems

2014 Gold Coast, Australia

This material is posted here with permission of the IEEE. Such permission of the IEEE does not in any way imply IEEE endorsement of any of BTH's products or services Internal or personal use of this material is permitted. However, permission to reprint/republish this material for advertising or promotional purposes or for creating new collective works for resale or redistribution must be obtained from the IEEE by sending a blank email message to pubs-permissions@ieee.org.

By choosing to view this document, you agree to all provisions of the copyright laws protecting it. 


\title{
Wireless Information and Power Transfer in an Underlay Cognitive Radio Network
}

\author{
Louis Sibomana $^{1}$, Hans-Jürgen Zepernick ${ }^{1}$, and Hung Tran ${ }^{2}$ \\ ${ }^{1}$ Blekinge Institute of Technology, SE-371 79 Karlskrona, Sweden \\ E-mail: $\{1 \mathrm{sm}, \mathrm{hjz}\} @$ bth.se \\ ${ }^{2}$ Information Technology Faculty, NIEM, 31-Phan Dinh Giot, Hanoi, Vietnam \\ E-mail: $\{$ tranhungemail\}@gmail.com
}

\begin{abstract}
In this paper, we consider a secondary network with simultaneous wireless information and power transfer in a spectrum sharing scenario. In particular, a secondary user (SU) transmitter communicates with multiple SU receivers (SU-Rxs) under the peak interference power constraint of the primary user receiver and the $\mathrm{SU}$ maximum transmit power limit. We apply a channel quality-based threshold and exploit opportunistic scheduling. Specifically, an SU-Rx with best channel condition among a set of SU-Rxs satisfying the threshold is scheduled for data transmission. The remaining SU-Rxs with channel condition below the threshold, harvest the radio frequency energy. Analytical expressions of the $\mathrm{SU}$ ergodic capacity, symbol error rate, throughput, and energy harvesting are obtained. An optimal threshold satisfying a given target outage probability is determined. Numerical results are provided to investigate the impact of different parameters on the secondary network performance.
\end{abstract}

\section{INTRODUCTION}

The cognitive radio network ( $\mathrm{CRN}$ ) concept is considered as a promising solution in improving radio spectrum utilization [1]-[3]. In a CRN, secondary users (SUs) share frequency bands with primary users (PUs) using spectrum access techniques such as underlay, overlay, and interweave schemes [2]. For the underlay CRN approach, the SU is allowed to transmit as long as the interference power at the PU receiver (PU-Rx) is maintained below a predefined interference threshold [1]. In this context, different transmit power strategies for the SU have been studied [4]-[7] to guarantee that no harmful interference is caused to the PU-Rx considering either peak interference power (PIP) or outage constraint at the PU-Rx.

On the other hand, wireless devices such as cellular phones and sensors are often energy-constrained due to the limited battery capacity. Recently, energy harvesting (EH) from the environment has drawn a lot of research interest [8]-[14] as a feasible solution for prolonging the lifetime of wireless communication devices. In particular, radio frequency (RF) signals radiated by the transmitters can be used as a source for wireless power transfer [10]. The integration of RF EH into wireless communication systems is motivated by the fact that RF signals can transport information and energy at the same time. Accordingly, the simultaneous wireless information and power transfer (SWIPT) system [8]-[14] is considered as a viable solution for supplying energy to low-power devices. It allows the same spectrum to be used for dual purposes while guaranteeing acceptable quality of service (QoS) of the user. The SWIPT system uses the same emitted electromagnetic wavefield to transport both energy that is harvested at the receiver, and information that is decoded by the receiver. Specifically, RF EH can be realized by converting the RF signal to a direct current (DC) signal using a rectenna (antenna integrated with a rectifier, e.g., diode). In [8], the receiver was assumed to perform information decoding (ID) and $\mathrm{EH}$ simultaneously from the same received signal. However, due to practical circuit limitations, the receiver cannot perform both ID and EH at the same time. Further in [9], two schemes have been proposed where the receiver either switches in time between ID and $\mathrm{EH}$, or splits the received signal to use one portion for ID and the remaining portion for EH. Moreover, the authors in [10], presented two receiver architectures, namely, separated and integrated information and energy receivers for a point-to-point wireless link. For both architectures, the rate-energy trade-off has been investigated. In [11]-[13], a multiuser multiple antenna SWIPT system has been studied where the achievable rate and $\mathrm{EH}$ have been analyzed. The work in [14] considered a harvest-then-transmit protocol where an access point broadcasts only wireless energy to all users in the downlink. Then, users send their independent information to the access point in the uplink. A maximum sum-throughput has been analyzed by jointly optimizing the time allocation to the downlink wireless energy transfer and the uplink wireless information transfer given a total time constraint [14].

Moreover, the system throughput can be improved by exploiting multiuser diversity (MUD) where the user with the best channel condition is opportunistically scheduled to receive/transmit at each time slot [15], [16]. To achieve MUD, the channel quality information of all users is required at the the scheduler or base station. In other words, each user has to measure its own channel state information (CSI) and feed it back to the scheduler. As the number of users becomes large, the throughput gain is improved but so is the feedback load. There is a trade-off between maximizing spectral efficiency and reducing feedback load. In [17], a scheduling technique known as selective MUD (SMUD) has been proposed for conventional wireless networks in which each user decides whether or not to feed back its CSI based on a predefined 
channel quality threshold. It is shown that SMUD can reduce feedback channel bandwidth requirements while providing a good portion of MUD gain. Note that the feedback load reduction is an important issue not only when considering conventional wireless networks but as well as in CRNs.

For information-only transfer, MUD in a spectrum sharing CRN has been considered in [18], [19] where the secondary network performance in terms of capacity [18], multiuser diversity gain, and ergodic throughput [19] have been analyzed. It is noted that the works in [18], [19] did not take account of the channel quality feedback condition. Furthermore, EH in CRN has been considered in [20], [21] where the SU transmitters (SU-Txs) opportunistically harvest RF energy from nearby PU transmitters (PU-Txs). It should be mentioned that the EH analysis in [20], [21] has been examined under an opportunistic spectrum access, i.e., an SU accesses the spectrum when the PU is idle. To the best our knowledge, no previous work studied the multiuser scheduling SWIPT with channel quality-based threshold in an underlay CRN.

In this paper, we investigate multiuser scheduling SWIPT for a downlink secondary network where an SU-Tx communicates with multiple SU receivers (SU-Rxs). The SUTx transmit power is subject to the PU interference power constraint and the SU maximum transmit power limit. Each SU-Rx performs either the ID or EH receiver model, i.e, it cannot decode the information data and harvest the RF energy at the same time. To reduce the feedback load while exploiting MUD gain in a SWIPT system, the SU-Rxs with channel condition above a predefined threshold are allowed to feed back their CSI to the SU-Tx. Further, the SU-Rx with best channel condition among the SU-Rxs satisfying the threshold is scheduled for the ID receiver model. The SU-Rxs below the threshold, harvest the ambient RF energy. On this basis, we derive the probability density function (PDF) of the received signal-to-noise ratio (SNR). Then, analytical expressions of the SU ergodic capacity, average symbol error rate (SER), average throughput, and average $\mathrm{EH}$ are obtained. We also determine the optimal threshold for feedback condition given a target outage probability. Numerical results are provided to investigate the impact of different parameters on the secondary network performance.

The rest of the paper is organized as follows. Section II describes the system model and scheduling mechanism. In Section III, analytical expressions of the considered performance metrics are derived. Section IV provides numerical results and discussions. Finally, Section V presents conclusions.

\section{SySTEM MODEL AND SCHEDULING MECHANISM}

\section{A. System model}

An underlay CRN system model is depicted in Fig.1 where the secondary network consists of an SU-Tx communicating with $N$ SU-Rxs whereas the primary network consists of a PU-Tx serving a PU-Rx. For a realistic scenario, SUTx can act as a base station in any cellular network or a femtocell access point in a wireless local area network [22]. In addition, the PU-Tx $\rightarrow$ PU-Rx link may be considered as an

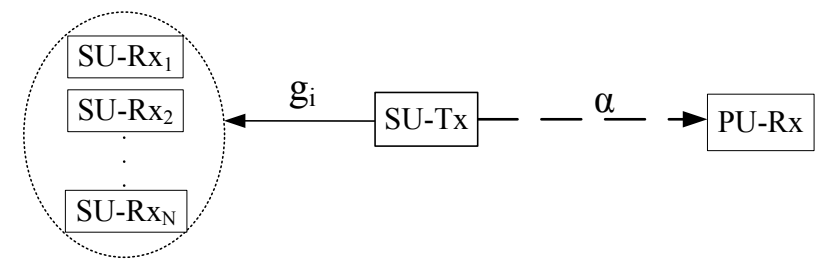

Fig. 1. Model of an underlay CRN system (Solid lines: Communication links, Dashed lines: Interference links).

uplink or downlink in a cellular network. The transmitter and receiver of each network has a single antenna and the available PU licensed bandwidth shared with the SU is normalized to unity. Moreover, we assume that the PU-Tx is far from the SU-Rx or the link PU-Tx $\rightarrow$ SU-Rx suffers from severe shadowing. Hence, the interference from PU-Tx to SU-Rx is not considered [5], [18].

Let $g_{i}, i=1,2, \ldots, N$ and $\alpha$ denote the channel power gains of the communication link SU-Tx $\rightarrow \mathrm{SU}^{\mathrm{Rx}} \mathrm{R}_{i}$ and interference link SU-Tx $\rightarrow$ PU-Rx, respectively. All channels are subject to independent Rayleigh fading and the channel gains remain constant during one time-slot but may vary independently through different transmissions. These channels are exponentially distributed random variables (RVs) with channel mean power denoted by $\Omega_{g_{i}}$ and $\Omega_{\alpha}$. We also define $\gamma_{i}$ as the received instantaneous SNR at the $i$ th SU-Rx expressed as

$$
\gamma_{i}=\frac{P_{s} g_{i}}{N_{0}}
$$

where $P_{s}$ is the instantaneous transmit power of the SU-Tx and $N_{0}$ is the noise power. As the PU and SU share the same frequency band, the SU-Tx must adjust its transmit power subject to a given interference power constraint to assure that the PU QoS is not violated.

\section{B. SU-Tx transmit power}

In an underlay CRN, the SU-Tx power control is mainly governed by the availability of the CSI of the SU-Tx $\rightarrow$ PURx link at the SU. When the PU PIP is considered [4], [5], [18], [19], perfect CSI of the primary link is assumed to be available at the SU-Tx in order for the SU to adapt its transmit power. In particular, the primary network CSI can be obtained through a band manager or directly fed back from the PU to the SU. In this case, the interference at the PU-Rx caused by the SU transmission is constrained by

$$
P_{s} \alpha \leq Q
$$

where $Q$ is the maximum acceptable level of interference that the PU-Rx can tolerate. Note that $\alpha$ must be perfectly known at the SU-Tx in order to satisfy (2). In practice, it is hard to obtain perfect CSI of the PU at the SU-Tx and costly in terms of feedback. When the CSI of the SU-Tx $\rightarrow$ PU-Rx link is imperfect, the SU-Tx may adjust its transmit power incorrectly which may cause higher interference to PU-Rx than the acceptable interference power $Q$. For the case of imperfect 
PU CSI at the SU-Tx (interference from the SU-Tx to the PURx exceeds the PU PIP constraint), a probabilistic constraint is considered with a certain low probability of error. Hence, the interference power constraint at the PU-Rx is reformulated as

$$
\operatorname{Pr}\left\{P_{s} \alpha>Q\right\} \leq \epsilon
$$

where $\epsilon$ is the tolerated error to satisfy (2). Here, the imperfect CSI is in the form of statistical channel knowledge, e.g., knowledge of the channel mean power $\Omega_{\alpha}$. This assumption is practical, since the collaboration between the PU and SU is limited. In addition, the SU-Tx can estimate $\Omega_{\alpha}$ as it is relatively stable. Furthermore, the average channel gain reduces the system feedback burden as it only requires infrequent updates [7].

By recalling that $\alpha$ is an exponentially distributed RV with mean $\Omega_{\alpha}$, we have

$$
\operatorname{Pr}\left\{P_{s} \alpha>Q\right\}=\exp \left(-\frac{Q}{P_{s} \Omega_{\alpha}}\right)
$$

Then, the SU-Tx transmit power is obtained from (4) as

$$
P_{s}=\frac{Q}{\Omega_{\alpha} \ln (1 / \epsilon)}
$$

As the transmit power cannot be infinite or very large in practice, the SU-Tx transmit power is also subject to the SU maximum transmit power limit denoted by $P_{\max }$ such that

$$
P_{s} \leq P_{\max }
$$

Based on (5) and (6), the received instantaneous SNR at the $i$ th SU-Rx given in (1) can be rewritten as

$$
\gamma_{i}=\min \left\{P_{s}, P_{\max }\right\} \frac{g_{i}}{N_{0}}
$$

In addition, we define $\gamma_{s}=P_{s} / N_{0}$ and $\gamma_{\max }=P_{\max } / N_{0}$ as the SU-Tx transmit SNR and maximum transmit limit SNR, respectively. By considering (5), (7) can be rewritten as

$$
\begin{aligned}
\gamma_{i} & =\min \left\{\frac{\gamma_{Q}}{\Omega_{\alpha} \ln (1 / \epsilon)}, \gamma_{\max }\right\} g_{i} \\
& =\mathcal{P} g_{i}
\end{aligned}
$$

where $\gamma_{Q}$ is the PU PIP SNR and $\mathcal{P}=\min \left\{\gamma_{s}, \gamma_{\max }\right\}$. Therefore, the received SNR depends only on the channel power gain of the SU-Tx $\rightarrow$ SU-Rx link at each transmission.

\section{Scheduling mechanism}

In the secondary network, we assume that the SU-Tx has perfect knowledge of CSI of each SU-Rx at the beginning of each transmission. Here, each SU-Rx estimates its CSI and then sends it to the SU-Tx through an error free feedback channel with negligible delay [15]. To reduce the bandwidth requirement for the feedback channel, the SU-Tx sets a threshold which may be given, for example, in terms of a target outage probability. The threshold is assumed to be the same for all SU-Rxs. Each SU-Rx tracks and compares its channel power gain to this threshold. Then, the SU-Rx feeds back its CSI to the SU-Tx only if the channel condition is above the given threshold, otherwise it remains silent. Note that this scheduling process has been considered in [17] for information-only transfer. In our ID receiver model, data information is transmitted only if there exist some acceptable SU-Rxs above the decoding threshold. In order to maximize the secondary network throughput, the SU-Tx selects the best $i$ th SU-Rx among the SU-Rxs satisfying the threshold condition, i.e., exploits Max-SNR scheduling [17]. For the remaining SU-Rxs below the threshold, the EH is performed. Thus, the scheduling process is formulated as

- ID receiver model: If $g_{i} \geq \mu_{t h}$, data is transmitted and the $i$ th SU-Rx is selected as

$$
j=\arg \max _{i \in\{1,2, \ldots, N\}}\left\{\gamma_{i} \mid g_{i} \geq \mu_{t h}\right\},
$$

where $\mu_{t h}$ is the feedback threshold condition.

- EH receiver model: If $g_{i}<\mu_{t h}$, the SU-Rxs below the threshold harvest the RF energy.

For the considered secondary network, we assume that the SU$\mathrm{Tx}$ is connected to a power supply (generator/battery) while the SU-Rx have no fixed energy source. In addition, each SURx has RF EH capabilities and therefore can harvest and store the wireless energy broadcast by the SU-Tx when the EH mode is active. Based on the channel conditions and predefined threshold, the SU-Rx decides when to switch between ID and EH modes.

\section{PERformanCE ANALYSis}

In this section, we derive the average $\mathrm{EH}$ for the $\mathrm{EH}$ receiver model. For the ID receiver model, analytical expressions of the ergodic capacity, average SER, and average throughput are also derived. To obtain these performance metrics, we first need to characterize the statistics of the output SNR in terms of the cumulative distribution function (CDF) and PDF of the channel output experienced by the scheduled SU-Rx.

For Rayleigh fading and recalling that $g_{i}$ is an exponentially distributed RV, the CDF and PDF of $g_{i}$ are given by

$$
\begin{aligned}
& F_{g_{i}}(x)=1-\exp \left(-\frac{x}{\Omega_{g_{i}}}\right) \\
& f_{g_{i}}(x)=\frac{1}{\Omega_{g_{i}}} \exp \left(-\frac{x}{\Omega_{g_{i}}}\right)
\end{aligned}
$$

Furthermore, we assume that the channel power gains have the same distribution and therefore, $\forall i: \Omega_{g_{i}}=\Omega_{g}$. This assumption applies to users that are essentially in a stationary environment, i.e., homogenous network [16], [17]. In addition, since each SU-Rx experiences the same channel statistics, the probability of getting access is equal for all SU-Rxs and hence, the scheduler is long-term fair.

\section{A. CDF and PDF of the output SNR}

Let $\gamma$ denote the output SNR of the scheduled SU-Rx. Based on the system scheduling of the ID and EH receiver models described above, the analysis must be conducted for two different regions, e.g., when all SU-Rxs remain silent and when at least one SU-Rx reports back its CSI. Recall that an SU-Rx can either perform ID or EH but not both at the same 
time. According to [17], the CDF of $\gamma$ represented by $F_{\gamma}(x)$ is expressed as

$$
\begin{aligned}
F_{\gamma}(x) & =\sum_{n=0}^{N}\left(\begin{array}{c}
N \\
n
\end{array}\right)\left[F_{g_{i}}\left(\mu_{t h}\right)\right]^{N-n} & & \\
& \times\left[F_{g_{i}}(x)-F_{g_{i}}\left(\mu_{t h}\right)\right]^{n}, & & g_{i} \geq \mu_{t h} \\
F_{\gamma}(x) & =\left[F_{g_{i}}\left(\mu_{t h}\right)\right]^{N-1} F_{g_{i}}(x), & & g_{i}<\mu_{t h}
\end{aligned}
$$

where $F_{g_{i}}\left(\mu_{t h}\right)=1-\exp \left(-\mu_{t h} / \Omega_{g}\right)$. Moreover, taking the derivative of (12) and (13) with respect to $x$, the PDF of $\gamma$ is obtained as

$$
\begin{aligned}
f_{\gamma}(x) & =\sum_{n=1}^{N}\left(\begin{array}{c}
N \\
n
\end{array}\right)\left[F_{g_{i}}\left(\mu_{t h}\right)\right]^{N-n} n f_{\gamma_{i}}(x) & & \\
& \times\left[F_{g_{i}}(x)-F_{g_{i}}\left(\mu_{t h}\right)\right]^{n-1}, & & g_{i} \geq \mu_{t h} \\
f_{\gamma}(x) & =\left[F_{g_{i}}\left(\mu_{t h}\right)\right]^{N-1} f_{g_{i}}(x), & & g_{i}<\mu_{t h}
\end{aligned}
$$

\section{B. Average energy harvesting}

For the EH receiver model and according to [10], [12], the amount of energy harvested at the SU-Rx is given by

$$
\mathrm{EH}_{i}=\eta \gamma_{i}
$$

where $0<\eta<1$ is a constant for the RF-to-DC energy conversion efficiency. Note that $\eta$ accounts for the loss in the energy transducer for converting the harvest energy to electrical energy. From (15) and (16), the average EH is obtained as

$$
\begin{aligned}
\mathrm{EH}_{\mathrm{avg}} & =\eta \mathcal{P} \mathbb{E}\left[g_{i}\right]=\eta \mathcal{P} \int_{0}^{\mu_{t h}} x f_{\gamma}(x) d x \\
& =\sum_{n=0}^{N-1}\left(\begin{array}{c}
N-1 \\
n
\end{array}\right)(-1)^{n} \exp \left(-\frac{n \mu_{t h}}{\Omega_{g}}\right) \\
& \times \eta \mathcal{P} \int_{0}^{\mu_{t h}} \frac{x}{\Omega_{g}} \exp \left(-\frac{x}{\Omega_{g}}\right) d x \\
& =\sum_{n=0}^{N-1}\left(\begin{array}{c}
N-1 \\
n
\end{array}\right)(-1)^{n} \exp \left(-\frac{n \mu_{t h}}{\Omega_{g}}\right) \\
& \times \eta \mathcal{P} \Omega_{g}\left[\begin{array}{c}
\left.1-\exp \left(-\frac{\mu_{t h}}{\Omega_{g}}\right)\left(1+\frac{\mu_{t h}}{\Omega_{g}}\right)\right]
\end{array}\right.
\end{aligned}
$$

where $\mathbb{E}[\cdot]$ denotes expectation and (17) is solved with the help of [23, eq. (3.351.7)].

\section{Ergodic capacity}

The secondary system capacity is defined as the maximum long-term achievable capacity, e.g., ergodic capacity over the time-varying fading channel. Based on the scheduling policy, the instantaneous achievable capacity in $\mathrm{bps} / \mathrm{Hz}$ at the scheduled $j$ th SU-Rx for the ID receiver model is expressed as

$$
C_{j}=\log _{2}\left(1+\mathcal{P} g_{j}\right)
$$

where

$$
g_{j}=\max _{i \in\{1,2, \ldots, N\}}\left\{g_{i} \mid g_{i} \geq \mu_{t h}\right\}
$$

The corresponding ergodic capacity is obtained as

$$
C_{\text {erg }}=\mathbb{E}\left[C_{j}\right]=\int_{\mu_{t h}}^{\infty} \log _{2}(1+\mathcal{P} x) f_{\gamma}(x) d x
$$

Substituting (14) into (20), and performing binomial expansions, we have

$$
\begin{aligned}
C_{\text {erg }} & =\sum_{n=1}^{N} \sum_{m=0}^{N-n} \sum_{k=0}^{n-1}\left(\begin{array}{c}
N \\
n
\end{array}\right)\left(\begin{array}{c}
N-n \\
m
\end{array}\right)\left(\begin{array}{c}
n-1 \\
k
\end{array}\right)(-1)^{m+k} \\
& \times \frac{n}{\Omega_{g}} \exp \left\{\frac{\mu_{t h}}{\Omega_{g}}[-m-(n-1)+k]\right\} I_{1}
\end{aligned}
$$

where

$$
I_{1}=\int_{\mu_{t h}}^{\infty} \log _{2}(1+\mathcal{P} x) \exp \left[-(k+1) \frac{x}{\Omega_{g}}\right] d x
$$

Then, applying integration by parts and L'Hôspital's rule in (22), and by using [23, eq. (3.352.2)], we obtain

$$
\begin{aligned}
I_{1} & =\frac{\Omega_{g} \log _{2}(e)}{k+1}\left\{\ln \left(1+\mathcal{P} \mu_{t h}\right) \exp \left[-(k+1) \frac{\mu_{t h}}{\Omega_{g}}\right]\right. \\
& \left.-\exp \left(\frac{k+1}{\mathcal{P} \Omega_{g}}\right) \operatorname{Ei}\left[-\frac{(k+1)}{\Omega_{g}}\left(\mu_{t h}+\frac{1}{\mathcal{P}}\right)\right]\right\}
\end{aligned}
$$

where $\operatorname{Ei}(z)=-\int_{-z}^{\infty} \frac{\exp (-t)}{t} d t$ is the exponential integral function.

\section{Average throughput}

The ergodic capacity obtained in (21) provides a theoretic idea on how the system achieves spectral efficiency for infinite number of modulation and coding schemes. However, the number of adaptive modulation and coding is limited for practical scenarios. For example, 3G (WCDMA/HSDPA) networks use two modulation schemes, namely, quadrature phase shift keying (QPSK) and 16-quadrature amplitude modulation (QAM). Therefore, it is important to investigate the system throughput for the considered secondary network with SWIPT. In particular, this paper considers a fixed modulation such as M-ary QAM [24], [25]. Then, the average throughput denoted by $\tau_{\text {avg }}$ for the ID receiver model is expressed as

$$
\tau_{\text {avg }}=b \int_{\mu_{t h}}^{\infty}[1-\operatorname{SER}(x)]^{L} f_{\gamma}(x) d x
$$

where $L$ is the packet length in bits and $b$ is the number of bits per symbol corresponding to the modulation scheme. In (24), $[1-\operatorname{SER}(x)]^{L}$ represents the packet error rate as a function of the instantaneous SER. It is hard to obtain an exact expression of (24) but an approximation for the SER is available for MQAM [24], [25] as

$$
\operatorname{SER}(x)=\theta \exp (-\delta x)
$$


where $\theta=0.2 b$ and $\delta=(1.6) /\left(2^{b}-1\right)$. Moreover, substituting (14) and (25) into (24), the average throughput is obtained as

$$
\begin{aligned}
\tau_{\mathrm{avg}} & =\sum_{l=0}^{L} \sum_{n=0}^{N} \sum_{m=0}^{N-n} \sum_{k=0}^{n}\left(\begin{array}{c}
N \\
n
\end{array}\right)\left(\begin{array}{c}
N-n \\
m
\end{array}\right)\left(\begin{array}{l}
n \\
k
\end{array}\right)(-1)^{l+m+k} \\
& \times \frac{n b(\theta)^{l}}{\Omega_{g}} \exp \left[-(m+n-1-k) \frac{\mu_{t h}}{\Omega_{g}}\right] \\
& \times \int_{\mu_{t h}}^{\infty} \exp \left[-x\left(\frac{k+1}{\Omega_{g}}+l \delta \mathcal{P}\right)\right] d x \\
& =\sum_{l=0}^{L} \sum_{n=0}^{N} \sum_{m=0}^{N-n} \sum_{k=0}^{n}\left(\begin{array}{c}
N \\
n
\end{array}\right)\left(\begin{array}{c}
N-n \\
m
\end{array}\right)\left(\begin{array}{l}
n \\
k
\end{array}\right)(-1)^{l+m+k} \\
& \times \frac{n b(\theta)^{l}}{k+1+l \delta \mathcal{P} \Omega_{g}} \exp \left[-\mu_{t h}\left(\frac{m+n}{\Omega_{g}}+l \delta \mathcal{P}\right)\right]
\end{aligned}
$$

\section{E. Average symbol error rate}

When data is transmitted, there is a possibility of error being caused to the system. The average SER denoted by $\overline{\text { SER }}$ is obtained by averaging the SER over the PDF of the scheduled SU-Rx. For the ID receiver model, the average SER is given by

$$
\overline{\mathrm{SER}}=\int_{\mu_{t h}}^{\infty} \operatorname{SER}(x) f_{\gamma}(x) d x
$$

Then, substituting (14) and (25) into (27), and after integration, we obtain

$$
\begin{aligned}
\overline{\mathrm{SER}} & =\sum_{n=1}^{N} \sum_{m=0}^{N-n} \sum_{k=0}^{n-1}\left(\begin{array}{c}
N \\
n
\end{array}\right)\left(\begin{array}{c}
N-n \\
m
\end{array}\right)\left(\begin{array}{c}
n-1 \\
k
\end{array}\right)(-1)^{m+k} \\
& \times \frac{\theta n}{k+1+\delta \mathcal{P} \Omega_{g}} \exp \left[-\mu_{t h}\left(\frac{m+n}{\Omega_{g}}+\delta \mathcal{P}\right)\right]
\end{aligned}
$$

\section{F. Optimal threshold choice}

The threshold choice has a significant and direct impact on the feedback condition. The higher the threshold, the lower the percentage of an SU-Rx to report its channel state. Here, the optimal threshold is obtained under a given target outage probability. By recalling that the threshold is identical for all SU-Rxs, the probability that $K$ out of $N$ SU-Rxs satisfy the threshold condition is given by

$$
\begin{aligned}
\mathrm{P}_{\mathrm{suc}} & =\operatorname{Pr}\{V=K\} \\
& =\left(\begin{array}{l}
N \\
K
\end{array}\right)\left[1-F_{g_{i}}\left(\mu_{t h}\right)\right]^{K}\left[F_{g_{i}}\left(\mu_{t h}\right)\right]^{N-K}
\end{aligned}
$$

where $V$ represents the number of SU-Rxs with channel condition greater or equal to the threshold. On the other hand, the probability that all SU-Rxs fail to exceed the predefined threshold is expressed in terms of outage probability as

$$
\begin{aligned}
\mathrm{P}_{\text {out }} & =\operatorname{Pr}\{V=0\}=\operatorname{Pr}\left\{g_{i}<\mu_{t h}, \quad \forall i \in\{1,2, \ldots, N\}\right\} \\
& =\left[F_{g_{i}}\left(\mu_{t h}\right)\right]^{N}=\left[1-\exp \left(-\frac{\mu_{t h}}{\Omega_{g}}\right)\right]^{N}
\end{aligned}
$$

since all SU-Rxs experience the same channel mean power. By inverting (30), the optimal feedback threshold $\mu_{t h}$ is obtained based on a required target outage probability as

$$
\mu_{t h}=\Omega_{g} \ln \left[\frac{1}{1-\left(\mathrm{P}_{\text {out }}\right)^{\frac{1}{N}}}\right]
$$

\section{NUMERICAL RESULTS}

In this section, we investigate the impact of different parameter settings on the secondary SWIPT system performance. In particular, we provide numerical examples based on MonteCarlo simulations together with analytical results. In the following, we set the PU-Rx tolerable error to satisfy (2) as $\epsilon=0.01$. In addition, we define $\mathrm{P}_{\text {out }}=\varepsilon_{s}$ as the $\mathrm{SU}$ required target outage probability for feedback threshold condition. Moreover, the channel mean power from the SU-Tx to the PU-Rx is characterized by $\Omega_{\alpha}=1 /\left(d_{1}\right)^{\nu}$ where $d_{1}$ is the normalized distance for the link SU-Tx $\rightarrow$ PU-Rx. Further, $\nu$ is the path loss exponent and is set to $\nu=4$ for typical nonline-of-sight propagation, e.g., highly shadowed urban area. We also define $\Omega_{g}=1 /\left(d_{2}\right)^{\nu}$ as the channel mean power of the link SU-Tx $\rightarrow$ SU-Rx where $d_{2}$ is the distance between SU-Tx and SU-Rx. It can be observed that in all examined scenarios, there is a good agreement between analytical results and simulations.

Fig. 2 shows the SU average EH versus PU PIP SNR $\gamma_{Q}$ for different numbers of SU-Rxs and different distances between SU-Tx and PU-Rx. In general, the average EH increases with the increase of the PU PIP SNR. This is expected since the increase of $\gamma_{Q}$ allows an increase of the SU-Tx transmit power following (5). Further, we can see that the distance between SU-Tx and PU-Rx has a significant impact on the SU average EH. Specifically, the average EH is low when the PU-Rx moves closer to the SU-Tx, e.g., from $d_{1}=1.2$ to $d_{1}=0.84$ for $N=4$ (number of SU-Rxs). In this case, the PU-Rx suffers stronger interference and hence, the SU-Tx must reduce its transmit power to satisfy the interference power constraint at the PU-Rx.

The effect of the number $N$ of SU-Rxs on the average EH is shown in Figs. 2 and 3. It can be observed that the average EH increases with the increase of $N$. For example, average $\mathrm{EH}$ is high by increasing $N=3$ to $N=6$ in Fig. 2 and EH generally increases for all scenarios in Fig. 3. This can be explained by the fact that having more SU-Rxs leads to the possibility of capturing more ambient RF energy. Also, the average EH increases when the energy conversion efficiency becomes more perfect $(\eta \rightarrow 1)$ as shown in Fig. 3 by increasing $\eta=0.5$ to $\eta=0.8$. In addition, Fig. 3 shows the impact of the distance between SU-Tx and SU-Rx on the average EH. We can see that the average $\mathrm{EH}$ is low when the SU-Rx is farther away from the SU-Tx, e.g., for the case of $d_{2}=0.84$ to $d_{2}=0.9$.

Fig. 4 shows the impact of the number $N$ of SU-Rxs, distance for the link SU-Tx $\rightarrow$ PU-Rx, and SU target outage probability on the SU ergodic capacity. Again, it is observed that the secondary network performance is improved when $N$ increases. For instance, the capacity is increased by increasing 


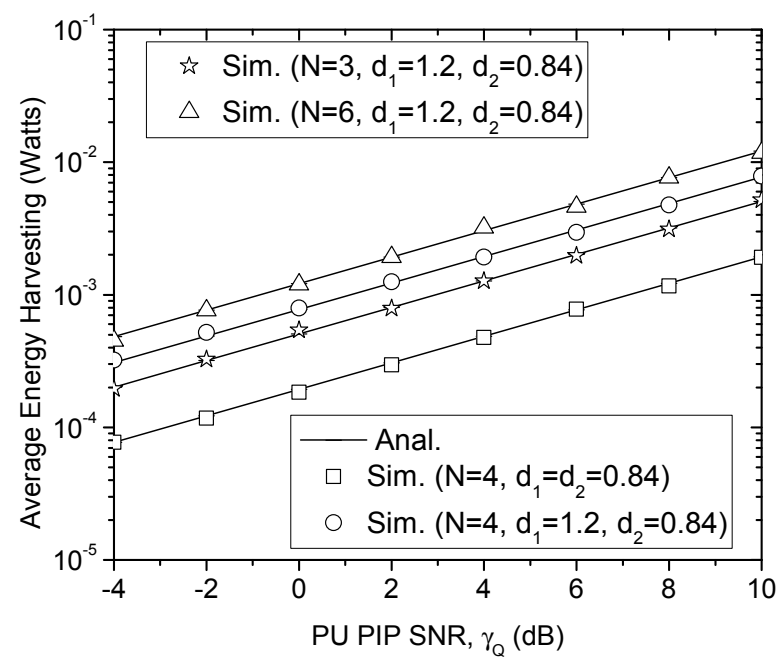

Fig. 2. Average EH versus PU PIP SNR $\gamma_{Q}$ with SU maximum transmit SNR $\gamma_{\max }=10 \mathrm{~dB}$, SU target outage probability $\varepsilon_{s}=0.01$, and SU energy conversion efficiency $\eta=0.5$.

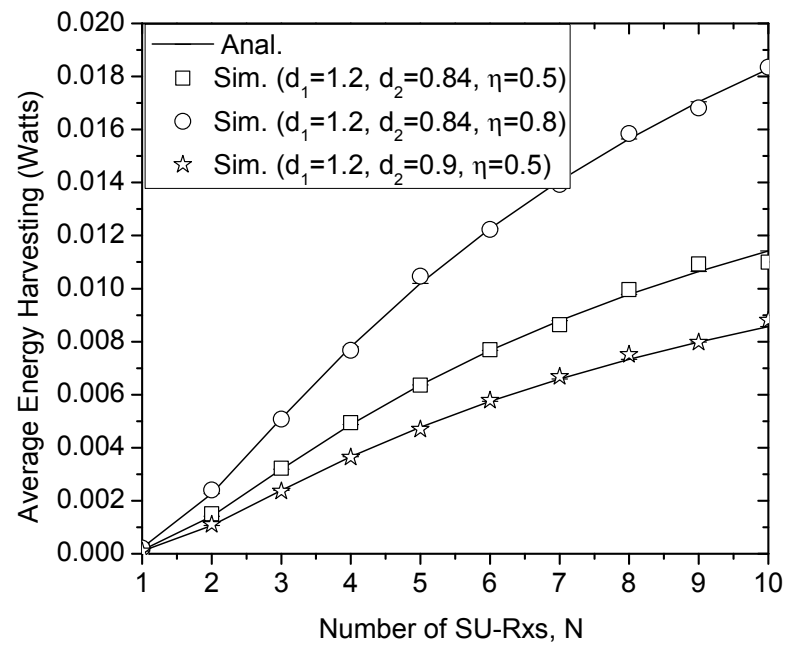

Fig. 3. Average EH versus number of SU-Rxs $N$ where PU PIP SNR $\gamma_{Q}=8 \mathrm{~dB}$, SU maximum transmit SNR $\gamma_{\max }=10 \mathrm{~dB}$, and $\mathrm{SU}$ target outage probability $\varepsilon_{s}=0.01$.

$N=1$ in Case 1 to $N=4$ in Case 2 . Here, the probability of getting good channel conditions is high (the feedback condition is satisfied) and hence the secondary network achieves better performance. As discussed previously in Fig. 2 for the $\mathrm{EH}$, the ergodic capacity is also decreased when the PU-Rx is closer to the SU-Tx, e.g., Case $3\left(d_{1}=0.84\right)$ compared to Case $2\left(d_{1}=1.2\right)$. Moreover, the effect of the SU target outage probability $\varepsilon_{s}$ is illustrated in Fig. 4. It is shown that the ergodic capacity is increased when the target outage probability is relaxed, e.g., $\varepsilon_{s}=0.03$ in Case 4 referred to $\varepsilon_{s}=0.01$ in Case 2 .

Figs. 5 and 6 illustrate the average SER and throughput, respectively. It is noted that the system parameter settings discussed for the ergodic capacity in Fig. 4 have the same effect on the average SER and throughput, respectively. For instance, Fig. 5 shows that the average SER is degraded when

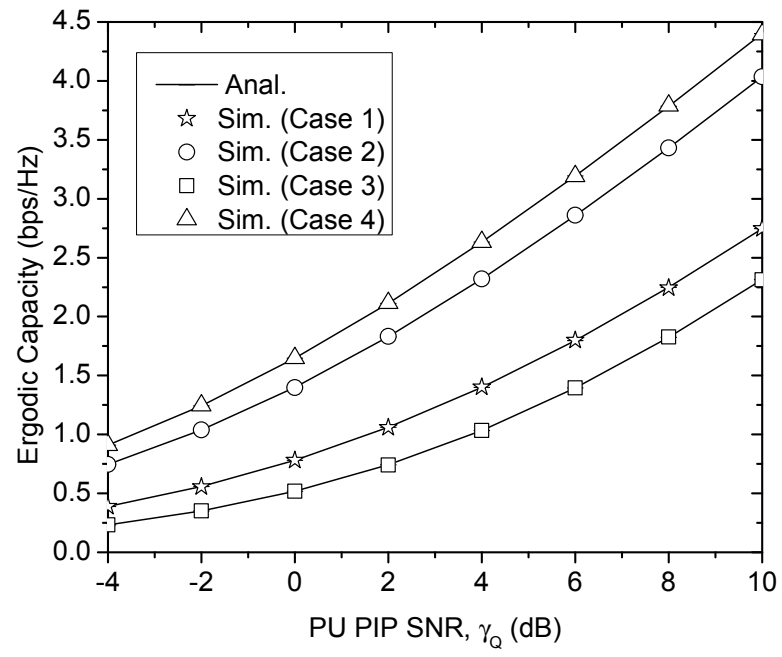

Fig. 4. Ergodic capacity versus PU PIP SNR $\gamma_{Q}$ with SU maximum transmit SNR $\gamma_{\max }=10 \mathrm{~dB}$ and $d_{2}=0.84$ (distance between SU-Tx and SU-Rx).

Case 1: $N=1, d_{1}=1.2, \varepsilon_{s}=0.01$;

Case 2: $N=4, d_{1}=1.2, \varepsilon_{s}=0.01$;

Case 3: $N=4, d_{1}=0.84, \varepsilon_{s}=0.01$;

Case 4: $N=4, d_{1}=1.2, \quad \varepsilon_{s}=0.03$

the distance between SU-Tx and PU-Rx becomes shorter, e.g., $d_{1}=1.2$ to $d_{1}=0.9$ for $N=6$ and BPSK modulation. It is also shown in Fig. 6 that by increasing the number of SU-Rxs, from $N=4$ to $N=8$ for BPSK and 8-QAM schemes, the average throughput is improved. Another interesting observation for SU throughput in Fig. 6 is that, for the low SNR region, BPSK modulation outperforms 8-QAM scheme. At high SNR region, e.g., $\gamma_{Q} \geq 8 \mathrm{~dB}$ with $N=8$, 8-QAM provides higher throughput. This can be confirmed by the fact that high order modulations provide better bandwidth efficiency but lower robustness against noise and fading.

It is observed that for high order modulations, some additional required SNR in $\mathrm{dB}$ is needed to achieve the same performance in terms of error as the low order modulations. The secondary system performance in terms of SER is improved for lower order of modulations. For example, we can see from Fig. 5 that the BPSK modulation provides low SER compared to 8-QAM scheme. If the performance metric of interest is the spectral efficiency, high order modulations become an advantage as shown in Fig. 6. Based on these results, we can state that the secondary network may adopt lower order modulation schemes since the secondary system operates in the low SNR region, i.e., the SU transmit power is limited by the imposed PU interference power constraint.

\section{CONCLUSiON}

In this paper, we have analyzed the secondary network performance with SWIPT under a PU PIP constraint. An optimal channel quality threshold for feedback load reduction has been obtained under a given target outage probability. Moreover, analytical expressions of the ergodic capacity, average SER, average throughput for the ID receiver model, and average $\mathrm{EH}$ for $\mathrm{EH}$ receiver model have been derived. The results illustrate 


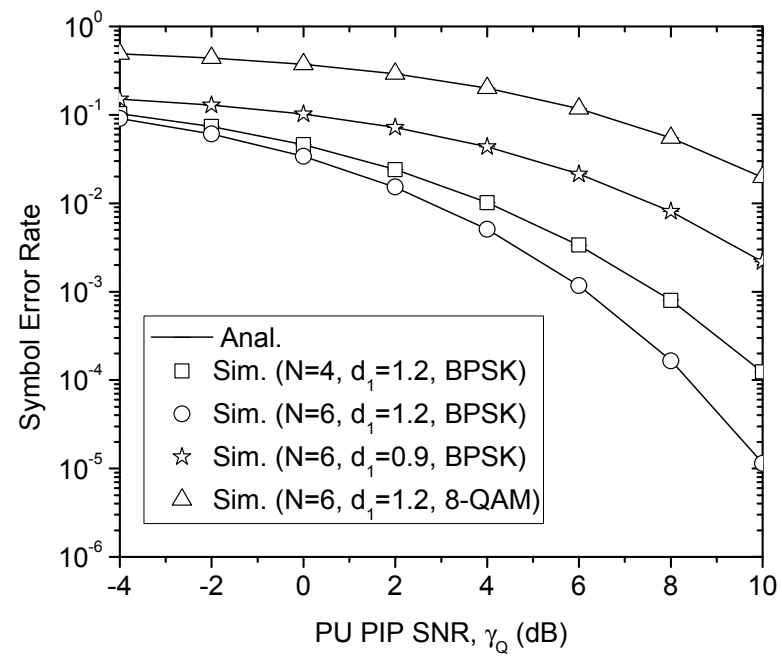

Fig. 5. Symbol error rate versus PU PIP SNR $\gamma_{Q}$ with SU maximum transmit SNR $\gamma_{\max }=10 \mathrm{~dB}, d_{2}=0.84$, and SU target outage probability $\varepsilon_{s}=0.01$.

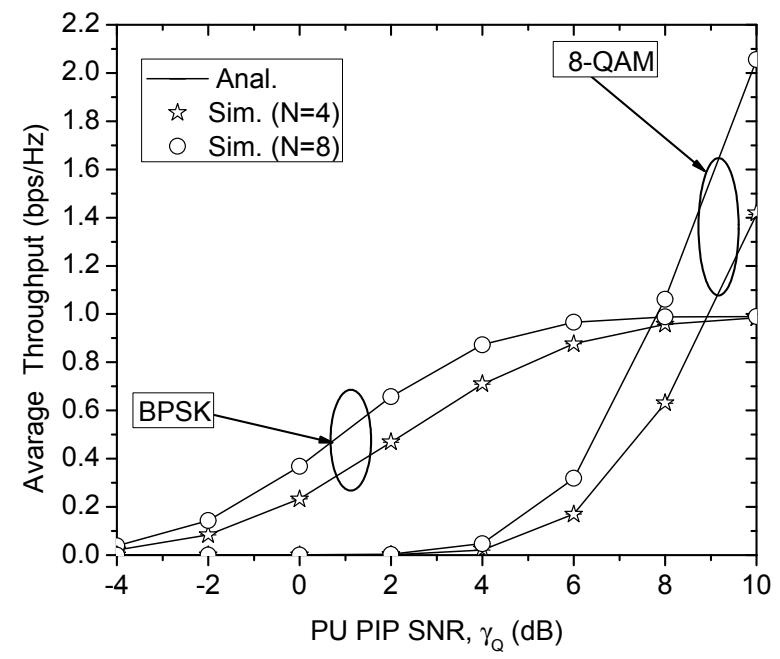

Fig. 6. Average throughput versus PU PIP SNR $\gamma_{Q}$ with SU maximum transmit SNR $\gamma_{\max }=10 \mathrm{~dB}, d_{2}=0.84, d_{2}=1.2$, SU packet length $L=50$ bits, and SU target outage probability $\varepsilon_{s}=0.01$.

that the number of SU-Rxs, SU target outage probability and distance between SU-Tx and PU-Rx have a significant impact on the secondary network performance. It is shown that the proposed multiuser scheduling SWIPT contributes to EH and achieves acceptable QoS for the user. The numerical examples also show the trade-off between theoretic capacity, throughput, and error performance for high and low order modulations.

\section{REFERENCES}

[1] A. Goldsmith, S. Jafar, I. Maric, and S. Srinivasa, "Breaking spectrum gridlock with cognitive radios: An information theoretic perspective," Proc. IEEE, vol. 97, no. 5, pp. 894-914, May 2009.

[2] M. G. Khoshkholgh, K. Navaie, and H. Yanikomeroglu, "Access strategies for spectrum sharing in fading environment: Overlay, underlay and mixed," IEEE Trans. Mobile Computing, vol. 9, no. 12, pp. 1780-1793, Dec. 2010.

[3] P. Pawelczak, K. Nolan, L. Doyle, S. W. Oh, and D. Cabric, "Cognitive radio: Ten years of experimentation and development," IEEE Commun. Mag., vol. 49, no. 3, pp. 90-100, Mar. 2011.
[4] R. Zhang, "On peak versus average interference power constraints for protecting primary users in cognitive radio networks," IEEE Trans. Wireless Commun., vol. 8, no. 4, pp. 2112-2120, Apr. 2009.

[5] X. Kang, Y.-C. Liang, A. Nallanathan, H. K. Garg, and R. Zhang, "Optimal power allocation for fading channels in cognitive radio networks: Ergodic capacity and outage capacity," IEEE Trans. Wireless Commun., vol. 8, no. 2, pp. 940-950, Feb. 2009

[6] X. Kang, R. Zhang, Y. Liang, and H. Krishna, "Optimal power allocation strategies for fading cognitive radio channels with primary user outage constraint," IEEE J. Sel. Areas Commun., vol. 29, no. 2, pp. 374-383, Feb. 2011

[7] P. J. Smith, P. A. Domochowski, H. A. Suraweera, and M. Shafi, "The effects of limited channel knowledge on cognitive radio system capacity," IEEE Trans. Veh. Techn., vol. 62, no. 2, pp. 927-933, Feb. 2013.

[8] L. R. Varshney, "Transporting information and energy simultaneously," in Proc. IEEE International Symposium Information Theory, Toronto, Canada, Jul. 2008, pp. 1612-1616.

[9] R. Zhang and C. K. Ho, "MIMO broadcasting for simultaneous wireless information and power transfer," IEEE Trans. Wireless Commun. vol. 12, no. 5, pp. 1989-2001, May 2013.

[10] X. Zhou and R. Zhang, "Wireless information and power transfer: Architecture design and rate-energy tradeoff," IEEE Trans. Wireless Commun., vol. 61, no. 11, pp. 4754-4767, Nov. 2013.

[11] K. Huang and E. Larsson, "Simultaneous information and power transfer for broadband wireless systems," IEEE Trans. Signal Process., vol. 61, no. 23 , pp. 5972-5986, Dec. 2013.

[12] X. Zou, R. Zhang, and C. K. Ho, "Wireless information and power transfer in multiuser OFDMA systems," IEEE Trans. Wireless Commun., vol. 13, no. 4, pp. 2282-2294, Apr. 2014.

[13] J. Xu, L. Liu, and R. Zhang, "Multiuser MISO beamforming for simultaneous wireless information and power transfer," in Proc. IEEE International Conf. Accoustics, Speech and Signal Processing, Paris, France, May 2013, pp. 4754-4758.

[14] H. Ju and R. Zhang, "Throughput maximization in wireless powered communication networks," IEEE Trans. Wireless Commun., vol. 13, no. 1, pp. 418-428, Jan. 2014.

[15] L. Yang and M. Alouini, "Performance analysis of multiuser selection diversity," IEEE Trans. Veh. Techn., vol. 55, no. 6, pp. 1848-1861, Nov. 2006.

[16] G. Song and Y. Li, "Asymptotic throughput analysis for channel-aware scheduling," IEEE Trans. Commun., vol. 54, no. 10, pp. 1827-1834, Oct. 2006.

[17] D. Gesbert and M.-S. Alouini, "How much feedback is multi-user diversity really worth?" in Proc. IEEE International Conf. Commun. Paris, France, Jun. 2004, pp. 234-238.

[18] T. W. Ban, W. Choi, B. C. Jung, and D. K. Sung, "Multiuser diversity in a spectrum sharing system," IEEE Trans. Wireless Commun., vol. 8, no. 1, pp. 102-106, Jan. 2009.

[19] R. Zhang and Y.-C. Liang, "Investigation on multiuser diversity in spectrum sharing based cognitive radio networks," IEEE Commun. Lett., vol. 14, no. 2, pp. 133-135, Feb. 2010.

[20] S. Park, H. Kim, and D. Hong, "Cognitive radio networks with energy harvesting," IEEE Trans. Wireless Commun., vol. 12, no. 3, pp. 13861397, Mar. 2013.

[21] S. Lee, R. Zhang, and K. Huang, "Opportunistic wireless energy harvesting in cognitive radio networks," IEEE Trans. Wireless Commun., vol. 12, no. 9, pp. 4788-4799, Sep. 2013.

[22] S. Al-Rubaye, A. Al-Dulaimi, and J. Cosmas, "Cognitive femtocell: Future wireless networks for indoor applications," IEEE Veh. Techn. Mag., vol. 6, no. 1, pp. 44-51, Mar. 2011.

[23] I. Gradshteyn and I. Ryzhik, Table of Integrals, Series and Products, 7th ed. Elsevier, 2007.

[24] S. Chunk and A. Goldsmith, "Degrees of freedom in adaptive modulation: A unified view," IEEE Trans. Commun., vol. 49, no. 9, pp. 1561-1571, Sep. 2001

[25] A. Kühne and A. Klein, "Throughput analysis of multiuser OFDMAsystems using imperfect CQI feedback and diversity techniques," IEEE J. Sel. Areas Commun., vol. 26, no. 8, pp. 1440-1450, Oct. 2008. 